\title{
Editor's Note: Special Issue on Big Data Analysis and Privacy
}

Published online: 2 February 2021

(C) Springer Science+Business Media, LLC, part of Springer Nature 2021

Journal of Signal Processing Systems gratefully acknowledges the ongoing editorial work of the scholar listed below on the special issue entitled "Big Data Analysis and Privacy."

Meikang Qiu

Columbia University.

USA

Publisher's Note Springer Nature remains neutral with regard to jurisdictional claims in published maps and institutional affiliations. 